\title{
Semiconductor Wireless Technology for Chronic Disease Management
}

\author{
L. Tarassenko and D.A. Clifton
}

Introduction: Over the last 50 years, healthcare expenditure has outpaced GDP growth by about $2 \%$ a year in most OECD countries. Chronic disease management accounts for $80 \%$ of this growth [1]. Chronic diseases (also known as long-term conditions) are defined by the World Health Organisation as "health problems that require on-going management for years or decades”. According to the figures for 2008, there are 15.4 million people in England with a chronic disease [2]; diabetes on its own takes up approximately a tenth of the NHS budget each year, a total exceeding £9bn [3]. Patients with chronic diseases account for $52 \%$ of all GP appointments, $65 \%$ of all outpatient appointments and $72 \%$ of all inpatient bed days [2].

In this review article, we will focus on those chronic diseases for which monitoring of one or more physiological variables can inform the management of the patient: asthma, diabetes, hypertension, Chronic Heart Failure (CHF), and Chronic Obstructive Pulmonary Disease (COPD). Each chronic disease has its own requirements, but we will attempt to make generic points which are broadly applicable to all such diseases.

Firstly, we will distinguish between active and passive monitoring. The former is well established as a component of self-management in chronic diseases such as type 1 diabetes or asthma, whereby the patient adjusts medication dosage according to the value of the measured variable. Passive monitoring is associated with more advanced chronic diseases, such as moderate-to-severe COPD or CHF. Such monitoring usually requires the use of wearable sensors for periods of time which may vary from minutes to days. The evaluation of passive monitoring for chronic disease management has so far been limited to pilot studies, however, and systematic reviews highlight the need for further research [4].

Active self-monitoring: Self-monitoring of peak expiratory flow (PEF) has been part of selfmanagement for asthma patients since the invention of the Wright peak flowmeter, a simple mechanical device, more than fifty years ago [5]. Similarly, regular blood glucose monitoring throughout the day has been a feature of self-management in type 1 diabetes, since the introduction of the first electronic glucometers thirty years ago [6]. For patients who require insulin injections (as is associated with type 1 diabetes or insulin-treated type 2 diabetes), the 
main aim of self-management is to take sufficient insulin to keep blood glucose levels down, whilst avoiding hypoglycaemia (low blood sugar). For asthma patients, the main aim of selfmanagement is the appropriate use of inhalers to deal with worsening symptoms, especially the use of steroids to cope with exacerbations as they begin to develop.

Self-monitoring may achieve improvements in outcome not only by judicious control of medication ("the expert patient") but also by providing advanced warning of adverse events (exacerbations for asthma patients and hypoglycaemia for diabetes patients). It can also give insights into factors that influence the course or progression of a disease and can support lifestyle changes. There is strong evidence showing the benefits of blood glucose testing to adjust insulin dose for people with type 1 diabetes in the context of a well-defined management plan [7]. The use of peak flow monitoring to improve outcomes for asthma has also been shown to be effective when embedded in an educational framework with patientdefined action plans [8]. Several studies (for example, Cappuccio et al. [9]) have shown that blood pressure control is better in patients with treated hypertension who self-monitor at home using a blood pressure monitor and inflatable cuff.

The frequency of self-monitoring will depend on the patient's chronic disease [10]. For example, patients with type 1 diabetes should measure their blood glucose (BG) levels three or four times a day, whereas hypertensive patients need only check their blood pressure once or twice a week. Patients with asthma should ideally monitor their peak flows in the morning and evening.

Self-monitoring in asthma or type 1 diabetes is the first step in a measure-evaluate-act feedback loop. Action consists in adjusting the dose of bronchodilator (delivered by a reliever inhaler) or corticosteroids (delivered by a preventer inhaler), or the dose of insulin, based on the measurement of PEF or BG and its evaluation. For asthma patients, evaluation involves an assessment of symptoms such as breathlessness, and for diabetes patients, food intake and physical activity need to be reviewed. This information is usually recorded on paper in a patient diary. The introduction of mobile phones with data storage and transmission capabilities, when the General Packet Radio Service (GPRS) network was switched on in 2002, enabled the implementation of an electronic patient diary on the phone. We developed at that time an integrated solution with an electronic BG (or PEF) meter linked to the 
patient's mobile phone via Bluetooth [11] [12] [13]. The physical separation between the electronic measuring device and the mobile phone is important as a fully integrated phone becomes a medical device and hence requires the relevant regulatory approvals.

Passive monitoring: The percentage of hospital bed days taken up by patients with one or more chronic diseases is putting a severe strain on healthcare systems. Often these patients oscillate back and forth between hospital and home or residential care. For example, 30\% of the COPD patients admitted to hospital in any given year for treatment of acute exacerbations are re-admitted at least once within the following year. The problem will only become worse with time unless the management of these patients in the community improves: by 2022, the proportion of people aged 65 and over in England will have risen to 10.8 million [2].

If we could decrease the number of unplanned hospital admissions, the economic burden of chronic disease management would be greatly reduced. The main objective of remote monitoring solutions ("telehealth") is to identify patient deterioration before it becomes sufficiently serious that the patient has to be admitted to hospital. A major obstacle to the widespread adoption of these solutions is the "human cost" of data collection. Many frail, elderly people find it difficult to continue with regular self-monitoring on a daily basis. Passive monitoring of vital signs with unobtrusive, wearable sensors is therefore an attractive option for them.

Heart rate, respiratory rate, arterial oxygen saturation $\left(\mathrm{SpO}_{2}\right)$, blood pressure, and temperature are the five vital signs which are measured non-invasively in hospital patients outside the Intensive Care Unit. They are recorded periodically by nursing staff on the general ward, but patients in more acute areas, such as the High-Dependency Unit or the Coronary Care Unit, are continuously monitored using bedside monitors. With a combination of wearable sensors and Bluetooth connectivity, it is now possible to acquire a subset of noninvasive measurements (heart rate, respiratory rate and arterial oxygen saturation) continuously in ambulatory patients [14] [15]. "Digital plasters” which exploit recent advances in semiconductor technology [16] [17] can now provide continuous monitoring and wireless transmission of vital-sign data for several days. As the reliability and robustness of this technology improve, its deployment into the home for remote monitoring of patients with chronic diseases will become more widespread. 
The low-power, silicon technology in digital plasters is designed to measure electrical currents or voltages, usually differentially. The electrocardiogram, recorded between two electrodes on the skin, provides a means of monitoring the patient's heart rate. The injection of a small, high-frequency current (typically $100 \mathrm{kHz}$ ) through the same pair of electrodes allows the respiratory rate to be derived from the changes in electrical impedance of the thorax during breathing. Skin temperature can also be sensed, although its relationship with core temperature depends on ambient temperature and perfusion at the site of measurement. Accelerometers can be micro-machined into the silicon substrate, to provide information on body position which can be integrated with vital sign data.

Optical measurements, which require different types of silicon sensors (such as light-emitting diodes and photodetectors), provide other non-invasive vital-sign data. The coloured substance in blood, haemoglobin, is also its carrier of oxygen, and the two common forms of the molecule, oxidised haemoglobin $\left(\mathrm{HbO}_{2}\right)$ and reduced haemoglobin $(\mathrm{Hb})$, have different optical spectra in the wavelength range from 500nm to $1000 \mathrm{~nm}$. Pulse oximeters measure the light transmission changes during the cardiac cycle through a body segment (such as a patient's finger, toe or earlobe) at two wavelengths [18], usually in the red (660 nm) and the near infra-red $(940 \mathrm{~nm})$. The cardiac-synchronous pulsatile waveform at either wavelength is known as the photoplethysmogram or PPG. The oxygen saturation $\left(\mathrm{SpO}_{2}\right)$ of the arterial blood in the body segment is derived from the ratio of the peak-to-trough amplitudes of the PPG waveform at the two wavelengths. The patient's heart rate (usually described as the pulse rate when obtained from the PPG) can be calculated from the interval between two consecutive peaks (or troughs) in the PPG waveform at one wavelength. Finally, respiratory rate can be estimated either from the breathing-synchronous modulation of the PPG waveform or from a measurement of heart rate variability over several respiratory cycles, typically one minute [19]. The heart rate increases during inspiration, a phenomenon known as respiratory sinus arrhythmia (RSA).

Self-monitoring of arterial oxygen saturation using a pulse oximeter is often used in the management of COPD patients at home [20]. This requires patients to use a finger probe connected to a pulse oximeter for a few minutes each day. While most pulse oximetry is performed using transmission-mode devices, in which light is passed through the tissues 
(often in a finger or earlobe) using a spring-loaded probe, an alternative method uses reflectance-mode devices. These latter, as the name suggests, measure the intensity of light reflected from tissues, allowing such devices to be constructed in "sticking plaster" configurations. With sicker patients, passive monitoring with reflectance-mode pulse oximetry would be more suitable, as these patients are more likely to tolerate an adhesive device, with the photodetector adjacent to the LED, for much longer than the spring-loaded probe of transmission-mode pulse oximetry. Reflectance-mode technology also provides a much greater choice of anatomical sites on which to position the probe (the chest or forearm, for example). It is a much less mature technology, however, mainly because the PPG signal can be affected by pressure disturbances acting on the probe, leading to inaccuracy in the measurement of $\mathrm{SpO}_{2}$ and pulse rate [21].

Future developments: The ideal digital plaster, or adhesive patch, for long-term passive monitoring of chronic disease patients would incorporate both electrical and optical measurements. All the vital signs except blood pressure could then be derived from a single device. Respiratory rate is perhaps the most important vital sign, as it is often the most significant early indicator of deterioration [22], but it is the most difficult to measure accurately. Electrical impedance signals are usually noisy and lead to inaccurate estimates of the patient's respiratory rate. Further estimates could be obtained from processing of the PPG waveform to extract the amplitude modulation and/or heart rate variability, and these independent estimates could then be fused to compute a more accurate value of respiratory rate. It should be added that no satisfactory method has yet been found to measure blood pressure non-invasively without the use of a cuff. Estimates based on the measurement of pulse wave velocity and/or pulse transit time have been found to be affected by several confounding factors [23].

Long-term passive monitoring requires a low background rate of data transmission (for example, once every minute) as most of the available power is consumed by the radiofrequency circuitry (usually Bluetooth or wi-fi) which links the processed sensor data to a mobile phone or wireless broad-band installation within the home. High-bandwidth data transmission should be reserved for "unusual or abnormal” vital-sign data. This relies on signal processing local to the sensor device to maximise the use of information: vital-sign data fusion can identify abnormal patterns early [24] [25], so that alert generation also 
triggers the transmission of the contents of a buffer in which the most recent high-bandwidth vital-sign data will have been stored.

Passive monitoring introduces stringent requirements on false alerting rates, as the patient is unaware of the abnormal data (as with vital-sign monitoring in hospital) and the carer responding to the alert is likely to be in a different location (unlike in-hospital patient monitoring). This makes reliable, passive vital-sign monitoring a much harder technical problem than active self-monitoring, hence the deliberate distinction in this review article.

Semiconductor technology is at the core of two new solutions which will increase the robustness of passive monitoring in the near-future: implantable sensors and non-contact PPG imaging. Implantable silicon sensors will soon be a viable option for managing patients with the most severe forms of chronic disease. Patients in the advanced stages of heart failure and/or pulmonary hypertension have a very restricted lifestyle unless the disease is controlled through medication. The most important measurement for titrating medication is pulmonary artery pressure (PAP). A number of implantable sensors for continuously measuring PAP are under development [26] [27], but one of the most promising approaches is a pressure-sensing surface acoustic wave device which is implanted during a simple catheterisation procedure when the patient is in hospital [28]. After the patient has returned home, PAP measurements can be made at any time (for example, once every five minutes), by interrogating the sensor by radio from a pocket-sized reader situated near the patient. This reader is similar to a mobile phone and it can be wirelessly linked to a remote carer.

Non-contact sensing of heart rate and $\mathrm{SpO}_{2}$ using webcams and ambient light, which has recently been demonstrated under controlled conditions, offers a radically new approach to the passive monitoring of patients in their homes. The possibility of measuring PPG signals remotely using a camera (rather than a probe attached to the finger, ear, or toe) was first reported in the scientific literature about five years ago [29] [30]. Verkruysse et al. [31] subsequently showed that PPG signals could be remotely acquired from the human face with ambient light as the source and a simple, digital, consumer-level camera as the detector placed more than 1m away from the subject. More recently, Poh et al. [32] [33] have used a webcam to record videos of facial regions in human volunteers, using independent component analysis to extract the PPG waveform from regions of interest in the image. This 
then allowed them to calculate both the heart rate and an estimate of respiratory rate from the RSA component of heart rate variability. Ambient light interference from fluorescent lights is a confounding effect, but non-contact sensing of vital signs could have a major impact on chronic disease management.

\section{Acknowledgements:}

The work described in this paper was funded by the NIHR Biomedical Research Centre Programme. Dr David Clifton is supported by the Wellcome Trust and EPSRC under grant number WT 088877/Z/09/Z.

\section{References:}

1. GSMA and McKinsey \& Company: 'mHealth: A new vision for healthcare', 2010. Available on line at: http://www.gsmworld.com/documents/mHealth_report.pdf. Accessed on 25th July 2011.

2. Department of Health. 'Raising the Profile of Long Term Conditions Care: A Compendium of Information', 2008. Available on-line at:

http://www.dh.gov.uk/prod_consum_dh/groups/dh_digitalassets/documents/digitalasset/dh_0 82067.pdf. Accessed on 25th July 2011.

3. 'The cost of diabetes'. Available on line at: http://www.diabetes.co.uk/cost-ofdiabetes.html. Accessed on 25th July 2011.

4. Nangalia, V., Prytherch, D.R., Smith, G.B.: 'Health technology assessment review: remote monitoring of vital signs--current status and future challenges’, Crit Care, 2010, 233

5. Wright, B.M., McKerrow C.B.: 'Maximum forced expiratory flow rate as a measure of ventilatory capacity’, BMJ, 1959, 2, pp. 1041-1047

6. Tattersall, R.B.: 'Home Blood Glucose Monitoring', Diabetologia, 1979, 16, pp. 7174

7. NICE Guidelines for the diagnosis and management of Type 1 diabetes in children, young people and adults. Guideline Number 15, July 2004

8. Gibson, P.G., Powell, H., Coughlan, J. et al.: 'Self-management education and regular practitioner review for adults with asthma', Cochrane Database Syst Rev, 2003, (1): CD001117

9. Cappuccio, F.P., Kerry, S.M, Forbes, L., Donald, A.: 'Blood pressure control by home monitoring: meta-analysis of randomised trials’, BMJ, 2004, 329:145 doi:10.1136/

bmj.38121.684410.AE 
10. Glasziou, P., Irwig, L., Mant, D.: 'Monitoring in chronic disease: a rational approach’, BMJ, 2005, 330: 644 doi: 10.1136/bmj.330.7492.644

11. Farmer, A., Gibson, O., Dudley, C., Bryden, K., Hayton, P., Tarassenko, L., Neil, A.: 'A randomized controlled trial of the effect of real-time telemedicine support on glycemic control in young adults with Type 1 diabetes, 2005, Diabetes Care, 28, pp. 2697-702

12. Ryan, D., Cobern, W., Wheeler, J., Price, D. and Tarassenko, L.: 'Mobile phone technology in the management of asthma. J Telemed Telecare, 2005, 11(Suppl. 1), pp. 43-46

13. Gibson, O.: 'Telemedicine for the Self-Management of Type 1 Diabetes', 2007, DPhil thesis, University of Oxford

14. Orphanidou, C., Clifton, D.A., Khan, S., Smith, M., Feldmar, J., Tarassenko, L.: 'Telemetry-based vital sign monitoring for ambulatory hospital patients', 2009. In: 31st Annual Int Conf IEEE Engineering in Medicine and Biology Society, Minneapolis, Minnesota

15. Harper, R., Donnelly, N., McCullough, I., Francey, J., Anderson, J., McLaughlin, J.A.D, Catherwood, PA.: 'Evaluation of a CE approved ambulatory patient monitoring device in a general medical ward’, 2010. In: 32nd Annual Int Conf IEEE EMBS, Buenos Aires, Argentina

16. Whelan, C.: 'The Doctor Is Out, but New Patient Monitoring and Robotics Technology Is In’, 2010. Scientific American, May 25, http://www.scientificamerican.com/article.cfm?id=patient-monitoring-tech

17. http://www.electronicsweekly.com/Articles/2010/01/27/47881/toumaz-trialselectronic-plaster-in-london-hospital.htm. Accessed on 25th July 2011.

18. Moyle, J.T.B.: 'Pulse Oximetry', 2002, $2^{\text {nd }}$ edition, BMJ Books, London

19. Meredith, D.J., Clifton, D., Charlton, P., Brooks, J., Pugh, C.W., Tarassenko, L.: 'Photoplethysmographic derivation of respiratory rate: A review of relevant physiology', Submitted to: J Med Eng Technol

20. National Collaborating Centre for Chronic Conditions. Chronic obstructive pulmonary disease. National clinical guideline on management of chronic obstructive pulmonary disease in adults in primary and secondary care. Thorax, 2004, 59(Suppl 1), pp. $1-232$

21. Dresher, R.: 'Wearable Forehead Pulse Oximetry: Minimization of Motion and Pressure Artifacts’, MSc Thesis, 2006, Worcester Polytechnic Institute

22. Seymour, C.W., Kahn, J.M, Cooke, C.R., Watkins, T.R., Heckbert, S.R., Rea, T.D.: 'Prediction of critical illness during out-of-hospital emergency care', JAMA, 2010, 304, pp. 747-754 
23. Douniama, C., Sauter, C.U., Couronne, R.: 'Blood pressure tracking capabilities of pulse transit times in different arterial segments: a clinical evaluation', Computers in Cardiology, 2009, 36, pp. 201-204

24. Tarassenko, L., Hann, A., Patterson, A., Braithwaite, E., Davidson, K., Barber, V., Young, D.: 'Multi-parameter monitoring for early warning of patient deterioration', Proc 3rd IEE Int Seminar on Medical Applications of Signal Processing, 2005, London, pp. 71-6

25. Tarassenko, L., Hann, A., Young D.: 'Integrated monitoring and analysis for early warning of patient deterioration’, British Journal of Anaesthesia, 97, pp. 64-8

26. Abraham, W.T., Adamson, P.B., Hasan, SA, Bourge, R.C. Pamboukian, S.V., Aaron, M.F., Raval, NY.: 'Safety and accuracy of a wireless pulmonary artery pressure monitoring system in patients with heart failure’, American Heart Journal, 2011, 161, pp. 558-566

27. Chow, E.Y., Chlebowski, A.L., Chakraborty, S., Chappell, W.J., Irazoqui, P.P.: 'Fully Wireless Implantable Cardiovascular Pressure Monitor Integrated with a Medical Stent', IEEE Trans Biomed Eng., 2010, 57, pp. 1487-1496

28. Toumazou, C., McLeod, C.N..: Implantable Surface Acoustic Wave Devices for Long Term Clinical Monitoring', 2007, US Patent Application US 20070282172

29. Wieringa, F.P, Mastik, F., Van Der Steen, A.F.W.: 'Contactless Multiple Wavelength Photoplethysmographic Imaging: A First Step Toward “SpO2 Camera” Technology', Annals of Biomedical Engineering, 2005, 33, pp. 1034-1041

30. Humphreys, K., Ward, T., Markham, C.: 'Noncontact simultaneous dual wavelength photoplethysmography: A further step toward noncontact pulse oximetry’, Rev. Sci. Instrum., 2007, 78, 044304

31. Verkruysse, W., Svaasand, L.O., Nelson, J.S.: 'Remote plethysmographic imaging using ambient light’, Optics Express, 2008, 16, pp. 21434-45

32. Poh, M.Z., McDuff, D.J., Picard, R.W.: 'Non-contact, automated cardiac pulse measurements using video imaging and blind source separation', Optics Express, 2010, 18, pp. 10762-10774

33. Poh, M.Z, McDuff, D.J., Picard, R.W.: 'Advancements in noncontact, multiparameter physiological measurements using a webcam’, IEEE Trans Biomed Eng., 2011, 58, pp. 7-11 